\title{
Prospects for Electroweak Physics at the LHC
}

\author{
Donatella Cavalli* \\ INFN Milano - via Celoria 16 - 20133 Milano - Italy \\ E-mail: 'donatelia. cavaili@imi.infn. ití
}

Abstract: The ATLAS and CMS experiments at the CERN Large Hadron Collider (LHC) will have a large discovery potential in the investigation of the nature of the electroweak simmetry breaking. The large event rates provided by the high luminosity LHC machine will also allow to perform precision measurements of Standard Model (SM) parameters. In this talk a few examples of the precise measurements that can be performed at the LHC are described (W and top mass measurements and Triple Gauge Couplings) and the SM Higgs searches, with particular emphasis on the low Higgs masses, and the measurement of the Higgs parameters are described.

\section{LHC running scenarios and cross sections}

The LHC will start running with a luminosity of $\sim 10^{33} \mathrm{~cm}^{-2} \mathrm{~s}^{-1}$ (low luminosity); this value should rise, during the first three years of operation, to the design luminosity of $10^{34} \mathrm{~cm}^{-2} \mathrm{~s}^{-1}$ (high luminosity). The first physics run is foreseen to start in August 2006.

Due to the expected high machine luminosity the production rates of many relevant physics processes are large (see Table $\left.\underline{1}_{1}^{\prime}\right)$. The non diffractive inelastic cross section is about $70 \mathrm{mb}$; this leads on average to a superposition of 2.3 (23) minimum bias events at low (high) luminosity on top of the hard collision. Precision measurements will therefore be mainly performed at low luminosity and in most cases they will be limited by systematics uncertainties.

In this LHC scenario, the main performance requirements of the detectors are: lepton measurement over the large range of $\sim 1 \mathrm{GeV}$ up to $\sim 5 \mathrm{TeV}$, excellent mass resolution (for masses around $100 \mathrm{GeV}: \sim 1 \%$ for leptonic decay channels and $\sim 10 \%$ for hadronic ones), calorimeter coverage up to $\eta \sim 5$, for a reliable transverse energy measurement and good forward jet tagging and very good identification of electrons, photons, taus and bjets. Moreover, there are three crucial parameters for precise measurements: the absolute luminosity, that should be measured at better than $5 \%$, the lepton energy scale, that should be measured at $0.1 \%$ in most cases and finally the jet energy scale, that should be known to $1 \%$.

\footnotetext{
${ }^{*}$ Speaker.
} 


\begin{tabular}{lccc}
\hline Process & $\sigma$ & events/s & Events/year \\
\hline $\mathrm{W} \rightarrow \mathrm{e} \nu$ & $15 \mathrm{nb}$ & 15 & $\sim 10^{8}$ \\
$\mathrm{Z} \rightarrow \mathrm{ee}$ & $1.5 \mathrm{nb}$ & 1.5 & $\sim 10^{7}$ \\
\hline $\mathrm{t} \overline{\mathrm{t}}$ & $800 \mathrm{pb}$ & 0.8 & $\sim 10^{7}$ \\
$\mathrm{~b} \overline{\mathrm{b}}$ & $500 \mu \mathrm{b}$ & $5 \times 10^{5}$ & $\sim 10^{12}$ \\
$\mathrm{QCD}$ jets $\left(p_{T}>200 \mathrm{GeV}\right)$ & $100 \mathrm{nb}$ & $10^{2}$ & $\sim 10^{9}$ \\
\hline$\tilde{\mathrm{g}} \tilde{\mathrm{g}}\left(m_{\tilde{\mathrm{g}}}=1 \mathrm{TeV}\right)$ & $1 \mathrm{pb}$ & 0.001 & $\sim 10^{4}$ \\
$\operatorname{Higgs}\left(m_{H}=120 \mathrm{GeV}\right)$ & $20 \mathrm{pb}$ & 0.02 & $\sim 10^{5}$ \\
\hline
\end{tabular}

Table 1: Cross sections, approximate number of expected events per second and order of magnitude of expected events per year for some physics processes at low luminosity $10^{33} \mathrm{~cm}^{-2} \mathrm{~s}^{-1}$.

\section{Measurement of the $\mathrm{W}$ mass}

At the time of the LHC start-up, the $\mathrm{W}$ mass will be known with a precision of $\sim 30$

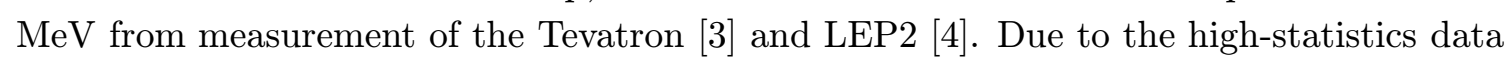
samples, the LHC should be able to improve on these results and therefore to provide more stringent tests of the underlying theory.

At hadron colliders, the $\mathrm{W}$ mass is obtained from the distribution of the $\mathrm{W}$ transverse mass. At the LHC, sixty millions of well measured $\mathrm{W} \rightarrow 1 \nu$ decays (where 1 is e or $\mu$ ) should be collected by each experiment in one year of data taking at low luminosity. The statistical error is therefore expected to be small $(<2 \mathrm{MeV})$; the systematic error will arise mainly from the Monte Carlo reliability in reproducing the data in terms of physics $\left(p_{T}{ }^{W}\right.$ spectrum, structure functions, $\mathrm{W}$ width, $\mathrm{W}$ radiative decays) and detector performance (absolute lepton energy scale, lepton energy resolution and detector response to the hadronic system recoiling against the W). Many of these uncertainties can be controlled in situ using the large $\mathrm{Z} \rightarrow \mathrm{ll}$ sample.

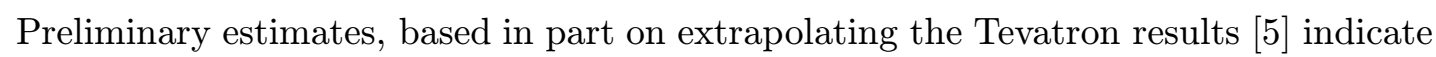
that, as it is the case today at the Tevatron, at the LHC the dominant uncertainty will originate from the calibration of the absolute lepton energy scale. For the $\mathrm{W}$ mass to be measured with a precision of $\sim 20 \mathrm{MeV}$, the lepton energy scale has to be known with a precision of $0.02 \%$, which represents the most serious challenge in this measurement. All other systematics are expected to be smaller than $10 \mathrm{MeV}$. By combining both ATLAS and CMS and both lepton channels it should be possible to measure the $\mathrm{W}$ mass with a total error of $15 \mathrm{MeV}$ in the first LHC phase at low luminosity.

\section{Measurement of the top mass}

At the LHC, top quark measurements will benefit from the large t $\bar{t}$ sample, so that not only the mass and the production cross section, but also branching ratios, couplings and rare decays can be studied in detail.

In the year 2006, the top mass should be known with a precision of $2-3 \mathrm{GeV}$ from measurements at the Tevatron $\left[\begin{array}{l}\overline{3} \\ \mathbf{n}\end{array}\right]$. At the LHC the best channel for the top mass measurement 
will be t $\overline{\mathrm{t}}$ production with one $\mathrm{W}$ decaying semileptonically and the other one decaying hadronically. The top mass will be determined from the hadronic part of the decay, while the leptonic part will be used to trigger the events and to suppress the background.

For one experiment, with $10 \mathrm{fb}^{-1}$, the statistical error is expected to be smaller than $0.07 \mathrm{GeV}$, the precision will be limited by the systematic error. An expected uncertainty of $1 \%$ on the absolute jet energy scale should translate into an uncertainty smaller than $1 \mathrm{GeV}$ on the top mass. The effect of the final-state gluon radiation is estimated to lead to an uncertainty of $\sim 1 \mathrm{GeV}$. Other sources of uncertainties (b-fragmentation, initial state radiation and backgrounds) are expected to be smaller. All together, a total uncertainty (most likely due to the knowledge of the physics) smaller than $1 \%$ should be achieved.

\section{Measurement of Triple-Gauge Couplings}

The study of Triple-Gauge Couplings (TGCs), i.e. couplings of the type $W W \gamma$ or WWZ, provides a direct test of the non-abelian structure of the SM gauge group. New Physics can show up as deviations of the five parameters: $g_{1}^{Z}, k_{Z}, k_{\gamma}, \lambda_{Z}, \lambda_{\gamma}$ from their SM values, which are zero for the $\lambda$ parameters and 1 for the $k$ and $g$ parameters. The LHC has a large potential for testing the TGCs because the sensitivity to the anomalous contributions ( $\lambda$ type in particular) is enhanced at high centre-of-mass energies.

TGCs will give rise to gauge boson pair production, e.g. $W \gamma, \mathrm{WZ}$ and $\mathrm{WW}$ production. Anomalous couplings can affect both the total cross section and the shape of differential distributions. As an example,

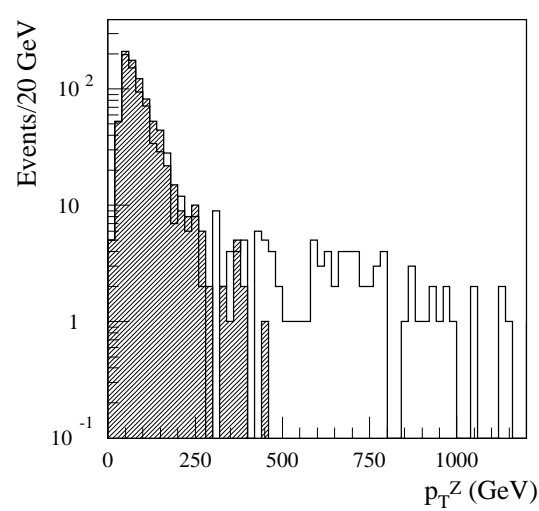

Figure 1: Reconstructed $p_{T}^{Z}$ in WZ events for SM (dashed) and with $\Delta g_{Z}^{1}=0.05$ (white) in ATLAS with $30 \mathrm{fb}^{-1}$. the reconstructed $p_{T}$ of the $\mathrm{Z}$ boson in WZ events is shown in Fig. II' for SM and in presence of non-standard anomalous couplings, that give an excess of events in the high- $p_{T}$ tail.

Assuming an integrated luminosity of $100 \mathrm{fb}^{-1}$, the expected statistical precision at 95\% CL for 1 experiment is found to be 0.034 and 0.040 for $\Delta k_{\gamma}$ and $\Delta k_{Z}$ respectively and 0.0038 for $\Delta g_{Z}^{1}$; the corresponding values for $\lambda_{\gamma}$ and $\lambda_{Z}$ are 0.0014 and 0.0028 respectively $(\Lambda=10 \mathrm{TeV})$. Systematic uncertainties from higher QCD corrections, structure functions and background are currently under study but should be small.

\section{The search for the SM Higgs boson}

The SM Higgs boson is produced at the LHC via gluon-gluon fusion, that is the dominant production channel for all masses, or via Weak Boson fusion WW/ZZ (WBF) and in associated $t \overline{\mathrm{t}} \mathrm{H}$ or $\mathrm{WH} / \mathrm{ZH}$ processes, that has a significantly smaller cross-section but has the additional signature from the decay of accompanying particles. The total production cross section is larger than $100 \mathrm{fb}$ up to $1 \mathrm{TeV}$. 
The search for a SM Higgs boson has been studied by ATLAS [i, [i] $]$ and CMS [i2] in various decay channels. The overall sensitivity for the discovery of the SM Higgs is shown in Fig.

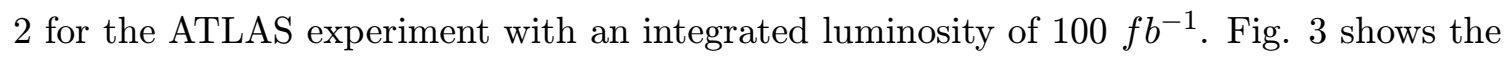
overall discovery potential combining ATLAS and CMS results for three different integrated luminosities. The Higgs boson discovery is possible over the full mass range from the LEP2 lower limit up to the $\mathrm{TeV}$ range already with $10 \mathrm{fb}^{-1}$. The different decay channels cover different regions, but over the full mass range more than one channel should be observed thus giving robustness to the discovery and hints to understand the nature of the signal.

The SM Higgs search is challenging in the low mass region. Here the two channels $(\mathrm{H} \rightarrow \gamma \gamma$ and $\mathrm{t} \overline{\mathrm{t}} \mathrm{H} \rightarrow \mathrm{t} \overline{\mathrm{tb}} \overline{\mathrm{b}})$ can be observed, however, the combination of both channels and of both LHC experiment is required to get a significance of $\sim 5$ with $10 \mathrm{fb}^{-1}$. Even though in this mass region the Higgs natural width is narrow, the backgrounds are relatively large and thus an excellent detector performance is required. The $\mathrm{H} \rightarrow \gamma \gamma$ decay mode requires high performance of the electromagnetic calorimeters in terms of mass resolution (photon energy resolution and direction measurement) and $\gamma /$ jet separation. Impact parameter measurements in the Inner Detector are crucial for the discovery of the $b \bar{b}$ decay mode.

In the intermediate mass region, excellent performance in terms of the identification, reconstruction and measurement efficiency of isolated leptons is required for the $\mathrm{H} \rightarrow \ell^{+} \ell^{-} \ell^{+} \ell^{-}$channel $\left[\overline{\hat{6}^{i}}\right]$.

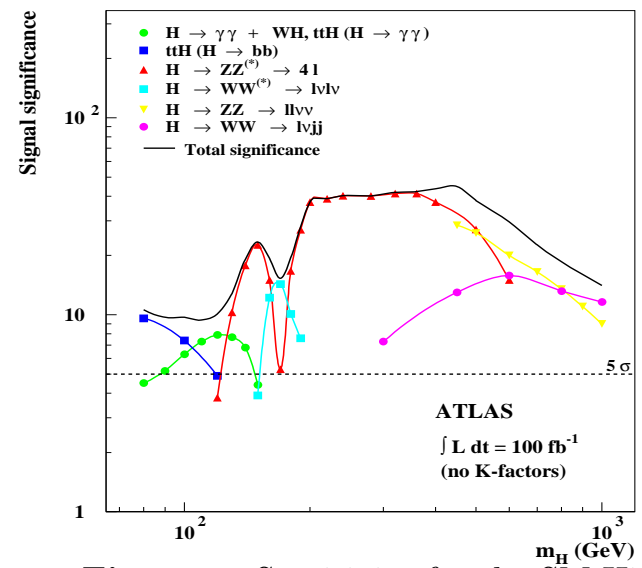

Figure 2: Sensitivity for the SM Higgs discovery, for ATLAS with $100 \mathrm{fb}^{-1}$. The different symbols are for different channels, the full line gives the combined significance.

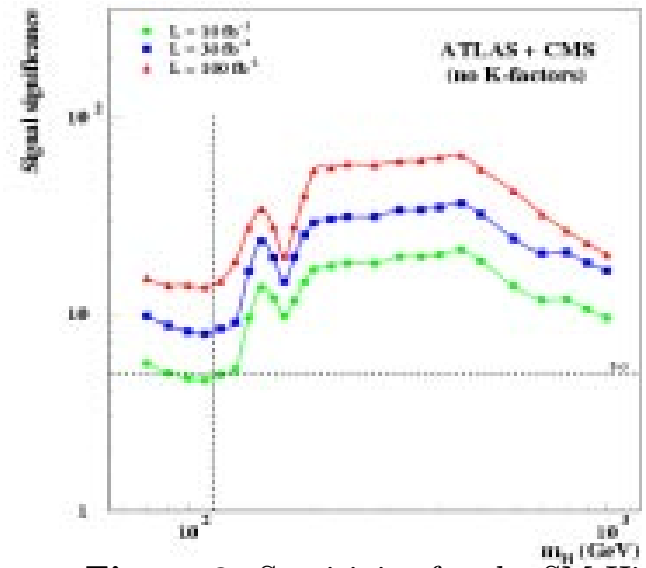

Figure 3: Sensitivity for the SM Higgs discovery, for ATLAS+CMS for 3 different integrated luminosities ( $\mathrm{L}$ is given per experiment).

The search for Higgs boson production via WBF, as originally proposed in $\left[\bar{T}_{i}\right]$, has also been studied. The results are not yet included in Fig. $\underset{2}{2}$ and $\overline{3}$. It has been found that WBF provides an additional significant discovery potential, although the production cross section is only the $20 \%$ of the total. In addition, it provides valuable information for the determination of the Higgs boson parameters, like couplings to bosons and fermions. The coupling to taus becomes also accessible, which is not possible using the dominant gluon fusion process, due to two distinctive signatures, the presence of two high $p_{T}$ forward jets and little jet activity in the central region, that provide a powerful tool for background rejection. 
Preliminary studies by both ATLAS and CMS in the channel qqH $\rightarrow$ qqWW $\rightarrow$ qql $\nu \mathrm{l} \nu$ confirm the WBF large discovery potential, also if the significance is worse than in [i].], due to ISR/FSR effects, jet calibration and lower efficiencies. A S/B ratio between 0.5 and 2.4 can be obtained for Higgs masses in $130-200 \mathrm{GeV}$, in ATLAS with $10 \mathrm{fb}^{-1}$. In the channel qqH $\rightarrow \mathrm{qq} \tau \tau$, with $30 \mathrm{fb}^{-1}$, a significance above 4 can be obtained in ATLAS for $m_{H}=120 \mathrm{GeV}$ considering all the lepton decay channels and a significance above 5 can be obtained in CMS for Higgs masses in 115-135 GeV, in the lepton-hadron channel.

\section{Measurement of Higgs Boson Parameters}

With an integrated luminosity of $300 \mathrm{fb}^{-1}$ ATLAS+CMS would measure the Higgs boson mass with a precision of $0.1 \%$ over the mass range $100-400 \mathrm{GeV}$, the Higgs boson width with a relative precision of $6 \%$ in the mass range $300-700 \mathrm{GeV}$, the Higgs boson production rates with a precision of $\sim 10 \%$ and several important couplings and branching ratios reaching a precision of $\sim 10-15 \%$ when using the same production mechanism and different decay channels and of $\sim 25 \%$ for measurements from different production mechanisms. These measurements should be able to give a first systematic investigation of Higgs properties.

\section{Conclusions}

The ATLAS and CMS experiments at the LHC should discover a Higgs boson (with 5 sigma) over the full mass range from the limit set by the previous machines up to the theoretical bound of $1 \mathrm{TeV}$ with $10 \mathrm{fb}^{-1}$. Different channels cover different mass regions, but over most of the mass range more than one channel should be observed, thus giving robustness to the discovery and hints to understand the nature of the signal. The large event samples, which will be produced at the LHC for many physics processes, will allow in addition to perform precision measurements of the parameters of the Standard Model.

\section{Acknowledgments}

I would like to thank the ATLAS Collaboration for giving me the opportunity to give this talk and for helpful discussions.

\section{References}

[1] ATLAS Collaboration, Detector and Physics Performance Technical Design Report, CERN/LHCC/99-15 (1999)

[2] CMS Collaboration, Technical Proposal, CERN/LHCC/94-38 (1994)

[3] D. Amidei and R Brock, FERMILAB-PUB-96/082 (1996)

[4] G. Altarelli, T. Sjostrand, F Zwirner, CERN 96-01 (1996)

[5] CDF Coll., Phys Rev. D52 (1995) 4784; D0 Coll., FERMILAB-PUB-97/328-E (1997).

[6] I. Puljak (CMS Collaboration), These de Doctorat Université Paris VI (2000)

[7] D. Zeppenfeld at al, Phys. Lett. B503,113 (2001) 